\title{
EXPERIMENTAL DETERMINATION OF THE ELECTRON-AVALANCHE AND THE ELECTRON-ION RECOMBINATION COEFFICIENT
}

\author{
G.J. ERNST and A.G. BOER \\ Department of Applied Physics, Twente University of Technology, \\ Enschede, The Netherlands
}

Received 14 April 1980

\begin{abstract}
The electron-ion recombination coefficient $\gamma$ and the avalanche coefficient $\delta=(\alpha-a) \cdot v_{\mathrm{d}}$, where $\alpha$ and $a$ are the ionization and attachment coefficients respectively and $v_{\mathrm{d}}$ the drift velocity of the electrons, have been experimentally determined in a self-sustained $\mathrm{CO}_{2}$-laser system $(1: 1: 3$ mixture) as a function of the $E / N$ value. For low voltages we found the expected decrease of the recombination coefficient for increasing $E / N$ values. However, it appears that for larger voltage the recombination coefficient increases sharply for increasing $E / N$ values. The measurements of $\delta$ show a much smaller value than expected from theoretical calculations. This must be explained by a lower value of the electronenergy distribution function for higher energies, which may be consistent with our measured high recombination probability for electrons having high energy.
\end{abstract}

\section{Introduction}

In order to predict the time behaviour of the gain and the output power of a $\mathrm{CO}_{2}$-laser system detailed knowledge is required of the time behaviour of the gas discharge. The electron density and the electron. energy distribution function are the most important parameters of a gas discharge. Their temporal behaviour is determined by the various loss and production processes, like ionization, attachment, electron-ion recombination, and all kinds of internal molecular ionizations.

Lowke et al. [1] predicted these characteristics by solving the Boltzmann transport equation for a $\mathrm{CO}_{2}$ $\mathrm{N}_{2}$-He mixture. Since the calculated electron-transport coefficients like drift velocity and characteristic energy in purc gases are in good agreement with experiment, it can be assumed that the relevant cross-sections and the relevant part of the distribution function, i.e. for energy values around the maximum of the distribution function, are reasonably accurate. However, the values of the attachment and ionization coefficients are very sensitive to changes in the tail of the distribution function and therefore a comparison between the theoretical and experimental values of the transport coefficients gives no indication of the accuracy of the ionization and attachment coefficients. Up to now no experimental values of these coefficients are available in the region of the stabilization voltage of the $\mathrm{CO}_{2}$. $\mathrm{N}_{2}$-He lasers, which is the most important region. For higher voltages good measurements have been perform. ed by electron-swarm methods [2]. However, these measurements have been performed with the gas mixture under a different condition than is usually the case for a real TEA laser system.

In this paper we will present experinental values for the difference between both coefficients for a $\mathrm{CO}_{2}: \mathrm{N}_{2}: \mathrm{He}=1: 1: 3$ mixture. The values are much smaller than theoretical predictions.

Concerning the electron-ion recombination coefficient $\gamma$ only very little experimental work has been done. Mills [3] performed measurements in an electron-beam system over a linited range of low $E / N$ values and Homann et al. [4] were not able to find an electron-density independent value of $\gamma$. Our measurements in a $\mathrm{CO}_{2}: \mathrm{N}_{2}: \mathrm{He}=1: 1: 3$ mixture have been performed up to an $E / N$ value of $5.3 \times 10^{-16} \mathrm{~V} \mathrm{~cm}^{2}$. For low values of $E / N$ we found a decreasing value of $\gamma$, whereas for larger $E / N$ values a sharp increase in $\gamma$ has been measured for increasing $E / N$ values. This increased $\gamma$ will provide for a smaller density of electrons hav. ing larger energies and can therefore explain the small values of $(\alpha-a)$ we found. 


\section{Experimental apparatus}

Our measurements have been performed with the laser system we recently developed and described in refs. [5] and [6]. The gap between the electrodes was $5 \mathrm{~cm}$ and the discharge length $40 \mathrm{~cm}$. The shape of the profiles are constructed according to Chang's paper [7] We used for his parameter $k$ the value 0.02 and for $v$ the value $\arccos (-k)$. We used a low-inductance 5 . stage marx generator, which could provide for a voltage of at least $200 \mathrm{kV}$. Our system has a built-in UV source, which produces a large amount of radiation because of the rapid current rise and the large area of the source. In this way a very high level of preionization is reached, resulting in a very homogeneous discharge without streamers and an excellent shot-to-shot reproducibility. Both are prerequisites for studying recombination and avalanche effects in the discharge. Since we constructed the system for low self-inductance, the connection between laser head and marx generator acts as a peaking capacitor over both electrodes. Because of the low self-inductance our system is very suitable for measurements on the electron-ion recombination, for it leads to high currents and thus to high electron densities.

The current measurements have been carried out by measuring the voltage over a resistance of $0.1 \Omega$, placed directly in series with the laser head. This resistance consisted of a row of 47 carbon resistors of 4.7 $\Omega$ each, connected in parallel. The response time of this current probe is less than $1 \mathrm{~ns}$.

Measuring the voltage over the profiles is a more difficult problem. We tried several methods and found a coppersulphate resistance divider to be the most suitable one for our purpose. The divider was connected directly to both laser profiles. It consisted of a methylmethacrylate tube having an inner diameter of $16 \mathrm{~mm}$ and a length of $30 \mathrm{~cm}$. At both ends a copper plug was placed for closing the tube and for the clectrical connections. At one end at a distance of $3 \mathrm{~mm}$ from the plug a probing copper pin, $1 \mathrm{~mm}$ in diameter, was fed through the tube wall. The tube was filled with an almost saturated coppersulphate solution. The total resistance was about $4 \mathrm{k} \Omega$. The signal from the copper pin was sent directly into a $50 \Omega$ cable. The attenuation, measured with a $200 \mathrm{~ns}$ pulse line charged to 20 $\mathrm{kV}$, turned out to be 213 times. The measurements showed that the attenuation is flat in time within a few percent and the response time is less than $1 \mathrm{~ns}$. Both the current and voltage signals are measured with a Tektronix Transient Digitizer 7912 AD, which is places in a Faraday cage. The long connecting cables gave an additional attenuation of $1.7 \%$.

\section{The recombination and avalanche coefficients}

The time behaviour of the electron density $n_{\mathrm{e}}$ in a $\mathrm{CO}_{2}-\mathrm{N}_{2} \cdot \mathrm{He}$ discharge, where charge neutrality is present, can be written as:

$\left(1 / n_{\mathrm{e}}\right) \mathrm{d} n_{\mathrm{e}} / \mathrm{d} t=v_{\mathrm{d}} \cdot(\alpha-a)-\gamma n_{\mathrm{e}}$,

where $v_{\mathrm{d}}$ is the drift velocity of the electrons, $\alpha$ and $a$ the ionization and attachment coefficients respectively and $\gamma$ the electron-ion recombination coefficient. Eq. (1) gives $\left(1 / n_{\mathrm{e}}\right) \mathrm{d} n_{\mathrm{e}} / \mathrm{d} t$ as a linear function of $n_{\mathrm{e}}$. So if $\left(1 / n_{\mathrm{e}}\right) \mathrm{d} n_{\mathrm{e}} / \mathrm{d} t$ and $n_{\mathrm{e}}$ can be experimentally determined, a graphic of the experimental points will give the quantities $\gamma$ and $\delta=v_{\mathrm{d}} \cdot(\alpha-a)$ from the tangent of the line and the intersection with the line $n_{\mathrm{e}}=0$ respectively. For the current it follows:

$I=n_{\mathrm{e}} e v_{\mathrm{d}} S$,

where $e$ is the electron charge and $S$ the cross-section of the discharge. Differentiating with respect to time gives:

$\frac{\mathrm{d} I}{\mathrm{~d} t}=e v_{\mathrm{d}} S \frac{\mathrm{d} n_{\mathrm{e}}}{\mathrm{d} t}+n_{\mathrm{e}} e S \frac{\partial v_{\mathrm{d}}}{\partial E} \cdot \frac{\mathrm{d} E}{\mathrm{~d} t}$

or

$\frac{1}{n_{\mathrm{e}}} \frac{\mathrm{d} n_{\mathrm{e}}}{\mathrm{d} t}=\frac{1}{I} \frac{\mathrm{d} I}{\mathrm{~d} t}-\frac{1}{v_{\mathrm{d}} \cdot G} \cdot \frac{\partial v_{\mathrm{d}}}{\partial E} \cdot \frac{\mathrm{d} V}{\mathrm{~d} t}$,

where $G$ is the electrode gap and $E$ and $V$ are the electric field strength and voltage between the electrodes respectively. In order to determine the unknown quantities $v_{\mathrm{d}}$ and $\partial v_{\mathrm{d}} / \partial E$ we used the time-of-flight measurements of Limbeek and Lucas [8]. The values of the drift velocity as well as the differential with respect to the electric field strength for a $\mathrm{CO}_{2}: \mathrm{N}_{2}: \mathrm{He}=$ 1:1:3 mixture as a function of the $E / N$ value are given in figs. 1 and 2 respectively. We used for $N$ the value $N=2.5 \times 10^{19} \mathrm{~cm}^{-3}$. From eqs. (2) and (4) $\left(1 / n_{\mathrm{e}}\right)$ $\mathrm{X} \mathrm{d} n_{\mathrm{e}} / \mathrm{d} t$ as well as $n_{\mathrm{e}}$ can be determined from measurements of the voltage and the current as a function of time. 


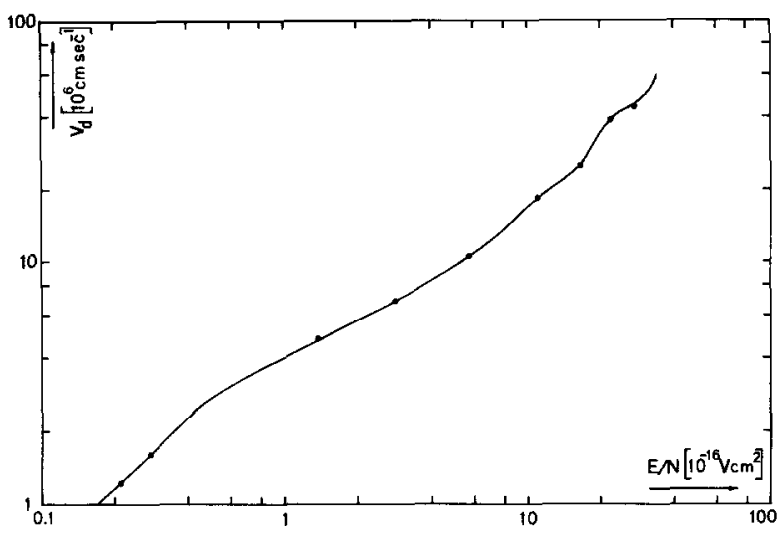

Fig. 1. The drift velocity as a function of the $E / N$ value in a mixture of one part $\mathrm{CO}_{2}$, one part $\mathrm{N}_{2}$ and three parts $\mathrm{He}$.

Because $\gamma$ and $\delta$ will be functions of the $E / N$ value, we performed current and voltage measurements for different charge voltage of the marxgenerator: 17.5 , $20,22,24,26$ and $28 \mathrm{kV}$. In all cases the capacitance of the marx generator was $0.16 \mu \mathrm{F}$. The first four measurements have been carried out with a total selfinductance of $1.06 \mu \mathrm{H}$ and the last two with a self-inductance of $0.63 \mu \mathrm{H}$. Then in every voltage picture the same voltage could be selected so that for every $E / N$ value six points could be collected where $I, V$, $\mathrm{d} I / \mathrm{d} t$, and $\mathrm{d} V / \mathrm{d} t$ are known. Usually a difficult problem in such a procedure is to find the time coincidence for the current and voltage measurements because they have been carried out one after the other. However,

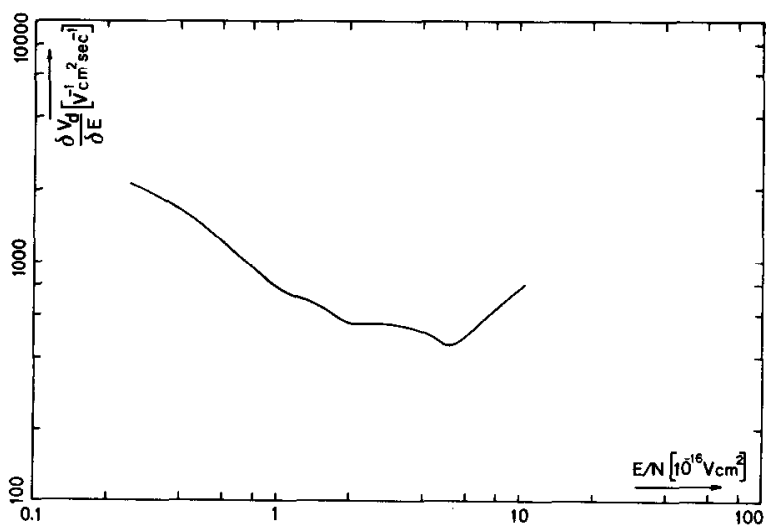

Fig. 2. The differential of the drift velocity to the electric field strength as a function of the $E / N$ value in a mixture of one part $\mathrm{CO}_{2}$, one part $\mathrm{N}_{2}$ and three parts $\mathrm{He}$. in our system the current rise and the consequent voltage drop takes place within 2 or $3 \mathrm{~ns}$ (for charge voltages of $20 \mathrm{kV}$ or higher), so that we were able to find the time coincidence of current and voltage within a few nanoseconds. For deducing the electron density from the current value the width of the discharge has to be known. We estimated the width of the discharge by scanning the laser output beam by a split and assuming the discharge width to be equal to the fwhm value of the output beam. We think that the assumption that the discharge width does not vary much in time during a pulse is reasonable. Fig. 3 shows the discharge width as a function of the marx generator charge voltage. In this way we could calculate from eq. (4) the relative change of the electron density with time as a function of the electron density. Fig. 4 shows our measurements for several $E / N$ values. As can be seen, the points lie on a straight line within the experimental error. The slope of this line is a direct measure of the electron-ion recombination rate. Extrapolating the line to $n_{\mathrm{e}}=0$ gives the value $\delta=(\alpha-a) v_{\mathrm{d}}$. In fig.5 the recombination-coefficient values determined from fig. 4 are plotted as a function of the $E / N$ value. It can be seen that for low values of $E / N$ the recombination coefficient decreases with increasing $E / N$ value. This behaviour is in accordance with the electron-beam measurements of Mills [3], who performed measurements in the low-voltage region. However, for large $E / N$ values an unexpectedly sharp increase in $\gamma$ has been found. Unfortunately it was not possible to extend the measurements to higher $E / N$ values. This would have been very interesting because this large in-

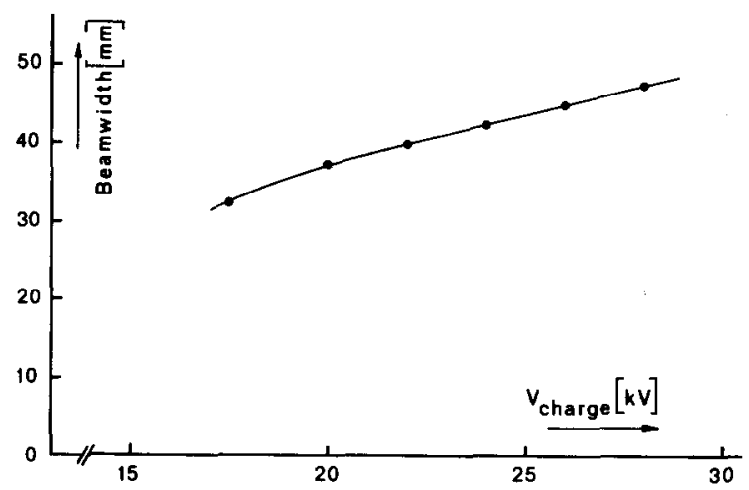

Fig. 3. The fwhm value of the output beam as a function of the charge voltage of the marx generator. 


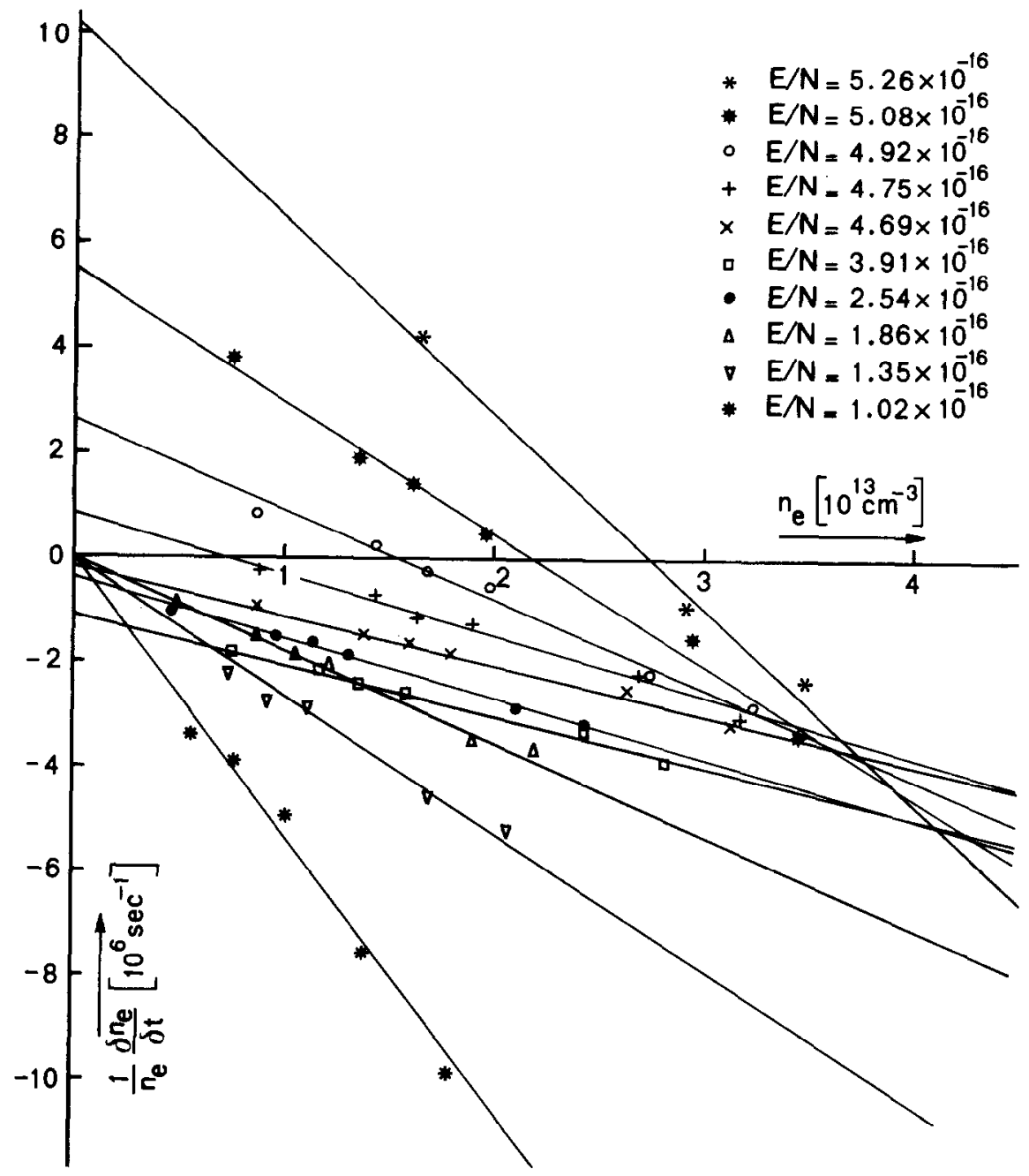

Fig. 4. The relative change of the electron density as a function of the electron density. The $E / N$ value of the discharge is used as a parameter.

crease in $\gamma$ will certainly affect the electron-energy distribution function for higher energies. Fig. 6 shows the values of $\delta$ as a function of $E / N$.

The $E / N$ value where the curve crosses the $\delta=0$ line is the socalled stabilization value of the discharge. We measured this value in a separate experiment by measuring the voltage of the laser under the condition that a large $200 \Omega$ resistor was connected in series with the laser. Then the electron density is so low that the voltage over the laser can be regarded as the stabilization voltage. Good agreement was found with our value determined from fig. 6 .

\section{Conclusions and discussion}

The measurements show that we have been able to determine the recombination coefficient rather accurately. The experimental error is lower than $10 \%$. For low values of $E / N$ our measurements can be compared with Mills' ones. Although they have different circumstances (electron-beam system and a somewhat lower pressure), our measurements agree remarkably well with his ones, so that we probably may conclude that self-sustained and EB systems do not differ with respect to their electron-ion recombination properties. 


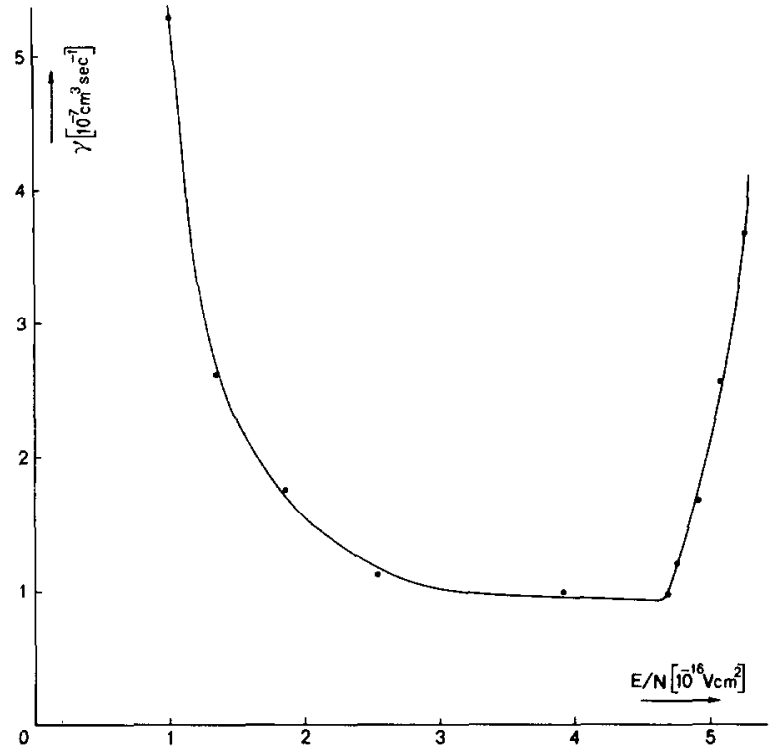

Fig. 5. The recombination coefficient as a function of the $E / N$ value for a $\mathrm{CO}_{2}: \mathrm{N}_{2}: \mathrm{He}=1: 1: 3$ mixture.

The sudden increase in the recombination coefficient above the $E / N$ value of $4.6 \times 10^{-16}$ is unexpected to us. The dominant ion in the discharge will be the one with the lowest ionization potential, i.e. $\mathrm{CO}_{2}$. Since the pressure is high, the majority of the ions will be present in the form of dimer ions and higher-order polymer ions of the form $\left(\mathrm{CO}_{2}^{+} \cdot n \mathrm{CO}_{2}\right)$. So the recombination will always be of the dissociative type. For dissociative recombination it is well-known that the coefficient decreases with increasing $E / N$ value. Prob. ably the increase at $4.6 \times 10^{-16} \mathrm{~V} \mathrm{~cm}^{2}$ is due to the opening of a new final state channel for recombination by way of a repulsive level of the polymer or dimer ion.

Comparing our results for $(\alpha-a) v_{\mathrm{d}}$ with calculations is more difficult. For $E / N=3.9 \times 10^{-16}$ we find a minimum valuc of $-1.1 \times 10^{6} \mathrm{~s}^{-1}$. The drift velocity being $8.2 \times 10^{6} \mathrm{~cm} / \mathrm{s}$, we find for $(\alpha-a)$ the value $-0.134 \mathrm{~cm}^{-1}$ or $(\alpha-a) / N=5 \times 10^{-21} \mathrm{~cm}^{2}$. Because this last value is mainly determined by attachment, we must conclude that our experimental attachment

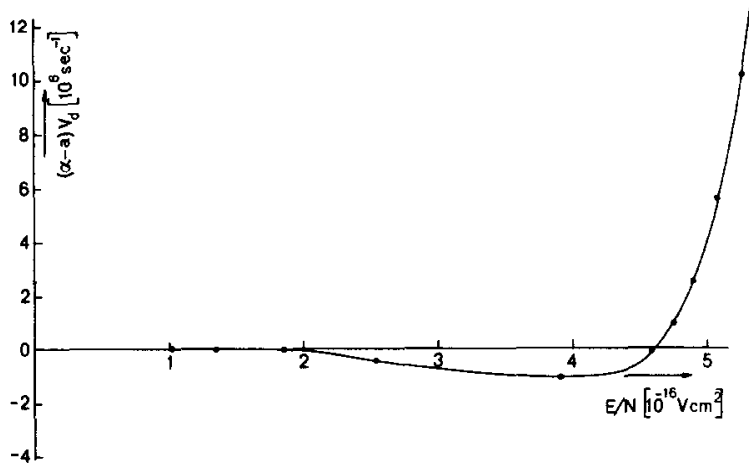

Fig. 6. The avalanche coefficient $\delta=(\alpha-a) v_{\mathrm{d}}$ as a function of the $E / N$ value for a $\mathrm{CO}_{2}: \mathrm{N}_{2}: \mathrm{He}=1: 1: 3$ mixture.

coefficient is about a factor of 4 smaller than theoretical predictions. About the same is probably true for the ionization coefficient; its value, however, is difficult to determine theoretically because it is very sensitive to the cross-section values of the ionization near the threshold energy. It is unlikely that the lower at. tachment and ionization coefficients are due to wrong values of the cross-sections. It is more likely that it is due to a lower value of the electron-energy distribution function for higher energies. This may be caused by the increased values of the recombination coefficient for high electron energies.

\section{References}

[1] J.J. Lowke, A.V. Phelps and B.W. Irwin, J. Appl. Phys. 44 (1973) 4664.

[2] C.S. Lakshminarasimha, J. Lucas, J.L. Moruzzi and I.J. Spalding, J. Phys. D: Appl. Phys. 9 (1976) 1727;

D. Kenneth Davies, J. Appl. Phys. 49 (1978) 127.

[3] C.B. Mills, J. Appl. Phys. 45 (1974) 1336.

[4] Ch. Homann, II. Hübner and W. Bötticher, Appl. Phys. Lett. 33 (1978) 417.

[5] G.J. Ernst and A.G. Boer, Optics Comm. 27 (1978) 105.

[6] G.J. Ernst and A.G. Boer, Optics Comm. 34 (1980) 221.

[7] T.Y. Chang, The Rev. Sci. Instr. 44 (1973) 405.

[8] J.W. Limbeek and J. Lucas, Solid State and Electron Devices 2 (1978) 161. 